\title{
Design and Development of Android Application for Doctors and Patients: Implications for Covid-19 Pandemic and Post Pandemic Uses
}

\author{
Ogunsola, K \& Akintoye, A.G \\ Africa Regional Centre for Information Science \\ University of Ibadan \\ Ibadan, Nigeria \\ E-mails: olukemi11@yahoo.com; mercyanu14@gmail.com \\ Phone Nos: +234-8068125011; +234-08155387707
}

\begin{abstract}
The anxiety brought by the Corona Virus Disease (COVID-19) pandemic caused a lockdown across the world to ensure social distance. This has pushed health care services far from the reach of many people, especially people with other ailments other than COVID-19. Outpatients become reluctant to visit healthcare centres to avoid the long waiting time to consult a doctor. There are also the problems of scheduling and rescheduling of appointments between patients and doctors, which has affected communication between them. Many hospitals have restricted patients' visits to hospitals and encouraged patients to communicate with their doctors via mobile phones. This has necessitated for the design and development of an android-based application called Medipal that improves consultation and appointment scheduling processes between doctors and patients, as well as personal health record keeping by patients. The application also enables patients to personally keep their health information, thereby involving them in their health and wellness. The overall performance of the mobile application was satisfactory as the evaluation showed that it enabled users to save time and cost during appointment scheduling and consultation. The app was deemed useful as majority accepted that it made them more aware of their health information. The interface of the app was easily navigated as users were able to perform operations with ease. Further research and development can be carried out as this study creates a platform upon which better systems can be built.
\end{abstract}

Keywords: Doctors, Healthcare, Medipal, Mobile Health Application, Mobile Technology, M-Health.

$2^{\text {th }}$ iSTEAMS Trans-Atlantic Multidisciplinary Conference Proceedings Reference Format

Ogunsola, K \& Akintoye, A.G (2020): Design and Development of Android Application for Doctors and Patients: Implications for Covid-19 Pandemic and Post Pandemic Uses. Proceedings of the 25th iSTEAMS Trans-Atlantic Multidisciplinary Virtual Conference, Laboratoire Jean Kuntzmann, Universite Laboratoire Jean Kuntzmann, Universite Grenoble, Alpes, France June - July, 2020. Pp 147-154

www.isteams.net/France2020. DOI - https://doi.org/10.22624/AIMS/iSTEAMS-2020N25P13

\section{INTRODUCTION}

Mobile Health (mHealth) is the delivery of healthcare services with the use of mobile technologies. The use of mobile technologies by healthcare professionals (HCPs) has transformed many aspects of clinical practice and assisted HCPs with many important tasks such as information and time management, health record maintenance and access, communication and consulting, reference and information gathering, patient management and monitoring, clinical decision-making, and medical education and training (Ventola, 2014). The intent of developing mHealth applications was to significantly improve healthcare and reduce cost by saving patients the extra costs associated with treatment like travel and other living expenses. 
It helps people manage their health, promote healthy living, and also helps them access to various important information when and where they are needed (GlobeNewswire, 2020). The need for mHealth applications cannot be left unattended to in health institutions in Nigeria, especially during the COVID-19 pandemic. The diffusion of ICT in healthcare addresses concerns like the late diagnosis of ailments, and delayed access to healthcare facilities. Even though mobile devices have reached far more people and settlements than basic amenities in Nigeria (United Nations, 2014), the healthcare systems still seem to lag in the implementation of the mobile health, as Nigeria has no policy or regulation guiding any aspect of electronic or mobile health (Salami 2015). A study by the Federal Ministry of Economic Cooperation and Development (2018), reveals that Nigeria was ranked in the $15^{\text {th }}$ position out of 17 countries in sub-Saharan Africa, with regards to their e-health regulatory readiness. The study further shows that Mauritius, Botswana and Seychelles occupied the first, second and third positions respectively.

There have been government initiatives as regards mHealth globally, this has however made it possible for the acceptance and adoption of mHealth apps in some countries across the globe (World Health Organisation, 2011). In South Korea, legislation was introduced to remove barriers in remote consultation in healthcare (MacGinnis\& Bennett, 2012). In Canada, the Canadian Medical Association (2015) provided guiding principles for physicians about how to assess a mobile health application for recommendation to a patient in the management of that patient's health, health care, and health care information.

\subsection{Significance of the Study}

The app will be of advantage to both doctors and patients who are the actual users of the app. The new app will ensure that patients find it easy to reach their doctors during the lockdown which is meant to ensure social distancing as well as after the lockdown. The app will also be of advantage to outpatients with ailments other than COVID-19. Medipal app will reduce crowds at healthcare centres which will subsequently remove frustration from patients. Patients may visit the clinics only when it is necessary. Besides, this application will avert users' distrust in mobile health applications due to lack of policies protecting its use. It will ensure that only doctors already obliged by the Code of Medical Ethics in Nigeria are engaged for consultation. The system provides a storage platform for the patient's health record which will engage patients in their health and wellness as they can securely store their personal health information and subsequently share it with doctors. The use of this application will bring about further growth and development in the health institutions as well as improve their reputation.

\section{RELATED WORKS}

Consultation time is the amount of time a patient spends seeing a medical doctor in a consulting room. The time spent depends on the nature of ailment and medical attention needed by the patient. It is important for any health facility to monitor its patients' waiting time, as it is an important indicator of the clinic's operational effectiveness and efficiency (Obulor and Eke, 2016). The Institute of Medicine (IOM) recommends that at least $90 \%$ of patients should be seen within 30 minutes of their scheduled appointment time (O'Malley, Fletcher, Fletcher and Earp, 1983). Scheduling appointments can be a difficult task for service providers that must meet the needs of both the customer and the service providers (O'Malley, Fletcher, Fletcher and Earp, 1983). Patient's waiting time refers to the time between the patient's arrival time and the service start time (Chen, Robielos, Palana, Valencia, and Chen, 2014). Scheduling patients' appointments is an important strategy to reduce patient's waiting time which subsequently improves the services of healthcare providers. 
A mobile health app, KompleteCare, was developed by the Society of Family Physicians of Nigeria (SOFPON) and a healthcare company called Sevenz Healthcare. The KompleteCare app is an android based application that creates a meeting point for doctors and patients online. Doctors are verified using their practising license before they are allowed to be consulted by users. Hudibia which was launched in 2016 is an app which allows users to search for doctors anywhere in the world, book medical appointments, have live consultations with doctors. The app creates a platform for patients to get medical services while doctors earn. LiveMD, Dokita247 and Talktomedoc are examples of appointment scheduling application where users register and consult registered doctors on the app from all over the world. Records may be defined as recorded information, irrespective of form or medium, received or created and maintained by establishments in pursuance of their statutory obligations of record keeping of any kind (Asunmo and Yaya, 2016). Dr Bridge app was invented in 2012 to help doctors keep electronic health records of their patients. The system is cloud-based as health records are stored in online servers. Stitch Manage app enables doctors to manage files containing patient's health information (Senkovska, 2017). Doctor On Demand, in addition to online consultation feature, has a patient's health record keeping feature which stores patients' health information securely.

In Nigeria, patients' records are normally stored in papers which can either be misplaced or destroyed (Oyegoke, 2013; Salami, 2015). There is no dedicated legal framework on data privacy protection generally or more specifically on data privacy for mHealth in Nigeria (Salami, 2015). Nigeria's 1999 Constitution, Code of Medical Ethics, and the Consumer Code of Practice Regulations only provide a broad and very limited effort to protect data privacy in the country (MacGinnis and Bennett, 2012). The Nigerian government has not implemented a policy to guide mHealth applications and its use; hence this might cause a downside in the prosperity of many existing and prospective mobile health projects. Due to lack of mHealth policy in Nigeria, the application developed from this study provides a platform for patients to consult only trusted doctors; that is, doctors that are licensed to have face-to-face contact with patients in the hospital and are covered by the Code of Medical Ethics of Nigeria.

For information provision relating to COVID-19 pandemic, The European Union's eHealth Network proposed the development of a mobile application to support contact tracing and warning in the EU's fight against COVID19 (eHealth Network, 2020). Likewise, Google and Apple jointly announced an initiative related to the use of the Bluetooth protocol to support contact tracing apps (Newsroom, 2020). These initiatives are still ongoing as at the time of this study. The World Health Organization provides a mobile app targeted at providing information to health workers battling the COVID-19 pandemic. The mobile app provides information on how health workers can protect themselves as they work, as well as furnish them with information on tools, training, and virtual workshops (World Health Organization, 2020). The app developed by the World Health Organization is developed for health workers, while Medipal is developed for both healthcare workers as well as patients.

\section{ANALYSIS AND DESIGN}

An interview session was conducted with 9 doctors and 21 patients in private and public healthcare centres in Ibadan, Nigeria to elicit information on challenges faced in the existing system and their probable needs from a new system. An analysis of the system showed that decisions on the patient's next visit for follow up or monitoring are always made by the doctor. Consultation in government and private hospitals involves physical interaction between the doctor and patient. From the first to subsequent visits, patients either come physically to the hospital or are visited at home by doctors. 
The safety and confidentiality of patients' health information is the sole responsibility of healthcare centres. For the new system, consultation and appointment scheduling are possible only when an invite has been sent by a party and accepted by the other party; this ensures mutual consent before users are enlisted for appointment and consultation. Users do not necessarily need to have contacts on their consultation list before they can start using the app. They can start managing health records immediately. The application also makes it possible for users to upload medical test results and other records in image format. The logical view of the application shows connections and data flow between various points of the application. The app consists of various activities and fragments. The activity is one of the app components which the app users interact with. Fragments are hosted by activities and they contribute their user interfaces to an activity.

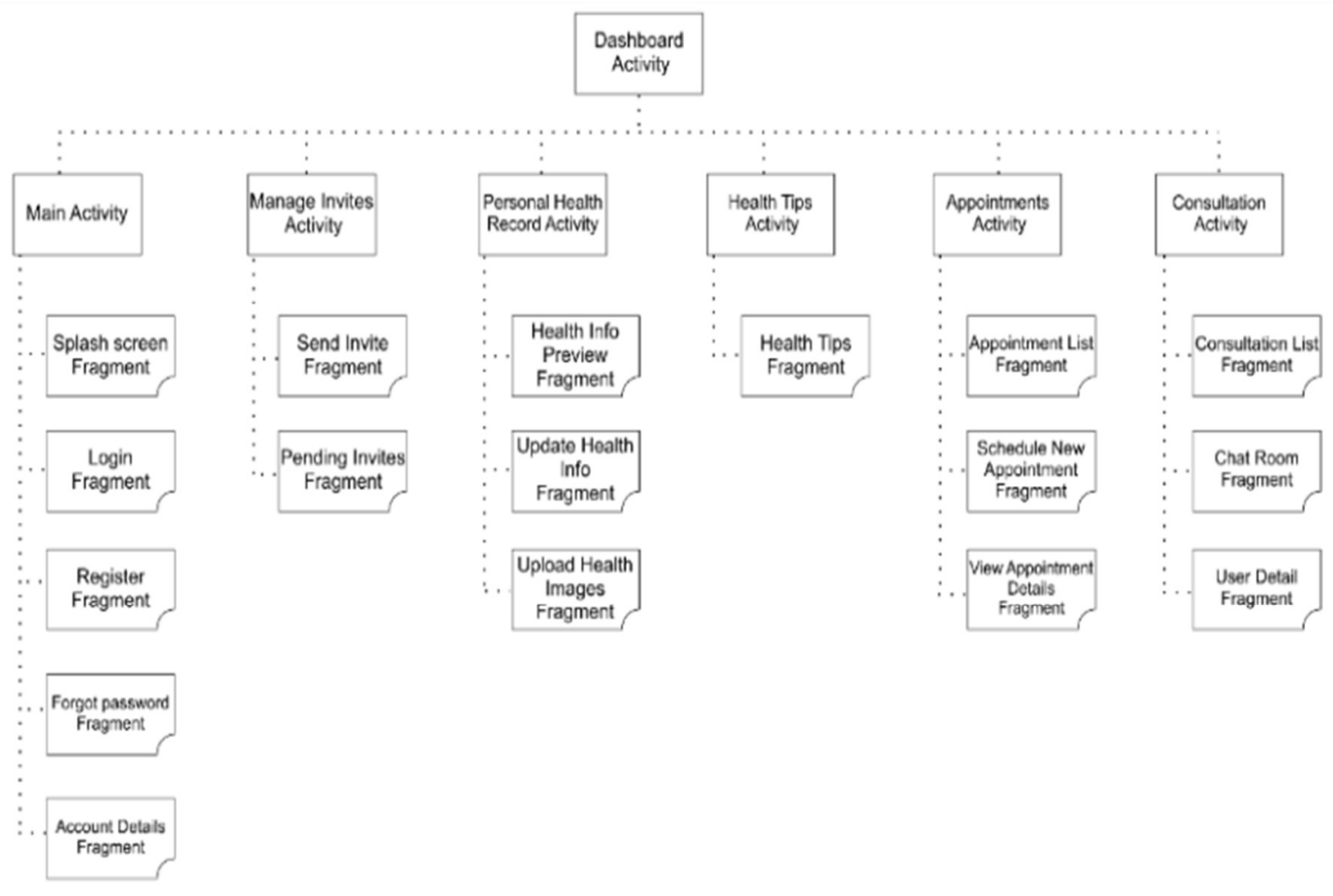

Figure 1: Activities and Fragments showing the Logical Flow of the App

Use case diagram helps to visualise the system's interaction with the environment through the use of actors and use cases. Use cases depict the tasks performed by the system users in different scenarios. They show a user's point of view during interaction with the system and vice versa. Figure 2 shows the app's use cases. 


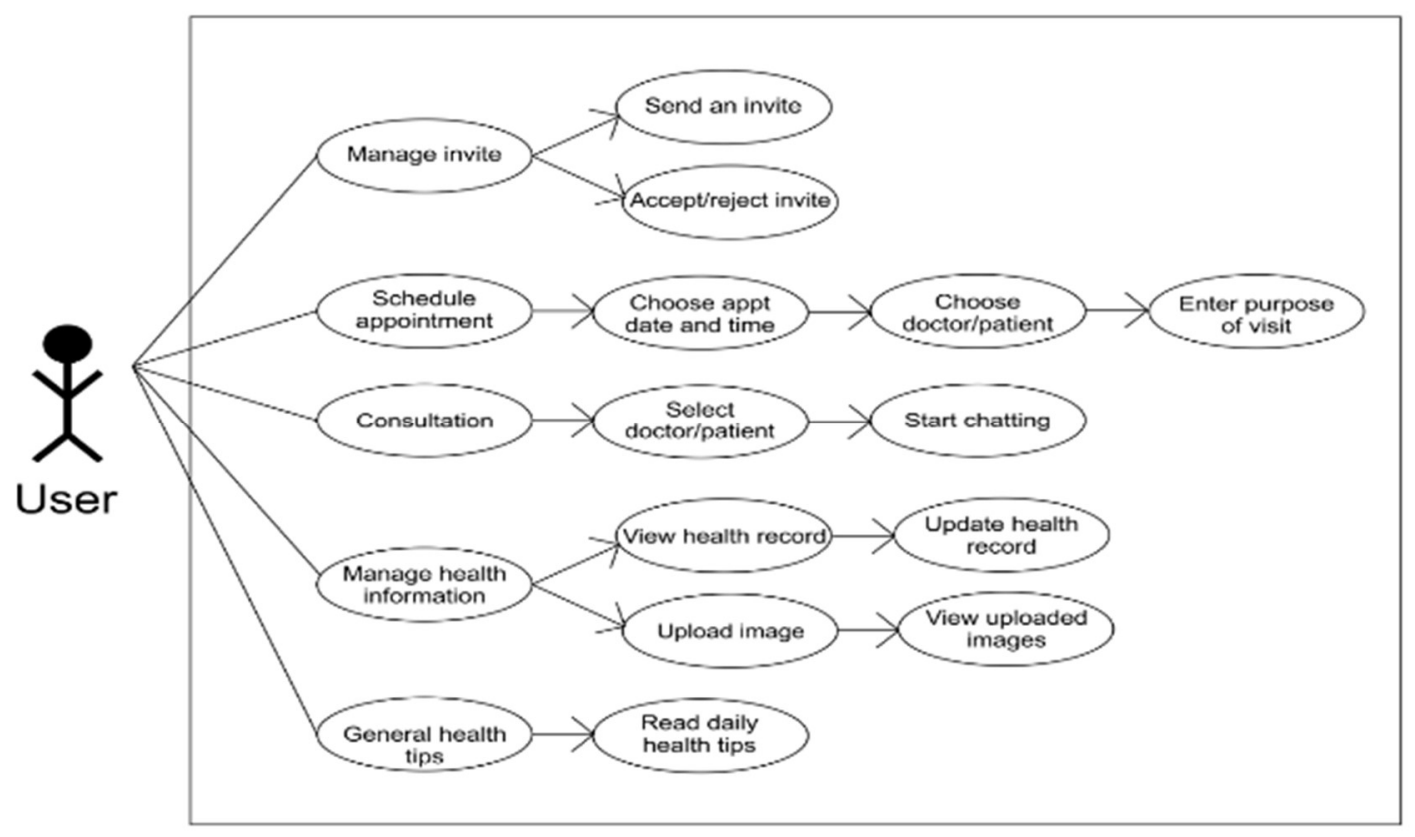

Figure 2: Use cases of App Users

The database used for this project's app is Cloud Firestore - a NO-SQL database service from Google's Firebase cloud service. Other tools from Firebase which this app uses are Authentication and Cloud Storage. Firebase is a backend-as-a-service mobile and web application development platform that provides a plethora of tools and service developers use (Stevenson, 2018). Firestore can store a wide range of data which include string, number, boolean, date and JSON. Firebase requires a developer to create a project on the dashboard and connect the project to the integrated development environment to initiate communication between the app and the online firestore server. Collections, documents and key-value pair data are automatically created when read and write operations are executed from the app java code.

\section{SYSTEM DEVELOPMENT}

The core software used in coding up the front and backend of the application was Android studio. The implementation of all logical description of the app was carried out on Android studio which is the official Integrated Development Environment (IDE) for Android application development. It has a unified environment where developers can develop apps for all Android devices. It has a flexible build system and features a rich emulator. Recent versions of the studio support Java and Kotlin as programming languages for developing Android apps. The JDK is a core software development package used for developing Java applications and applets. The JDK is a package of tools for developing Java-based applications. It has a sub-package called the Java Runtime Environment (JRE) which enables the actual execution of developed applications. The business logic of Medipal was developed using Java and XML. The program was modularized by separating the overall functionalities of the program into interdependent and interchangeable modules. Each of these modules was built, tested and debugged to separately execute only one aspect of the desired software functionality. 
The app developed in this project was decomposed into smaller and manageable components to allow easy planning, coding, debugging and testing. The modules include Authentication, Invite User, Appointment Scheduling, Online consultation, Personal Health Record and Health Tips. The health tips comprised general health tips as well as information relating to how people can stay safe from COVID-19 Pandemic and the contact of Nigeria Centre for Disease Control (NDDC).

\section{IMPLEMENTATION}

After efficient coding and testing of the application, the source code and application resources were cleaned and built to generate a newly signed bundle from the Android Studio integrated development environment. The installation process can be implemented using different approaches. The commonly used approaches include parallel approach, direct cut-over, single location and phased approach. Since the installation approach or a combination of installation approaches employed in the system installation depends on how easily targeted users of the application can adjust to the new transition, the direct and parallel approach was best deemed fit for this study, hence, the combination was adopted.
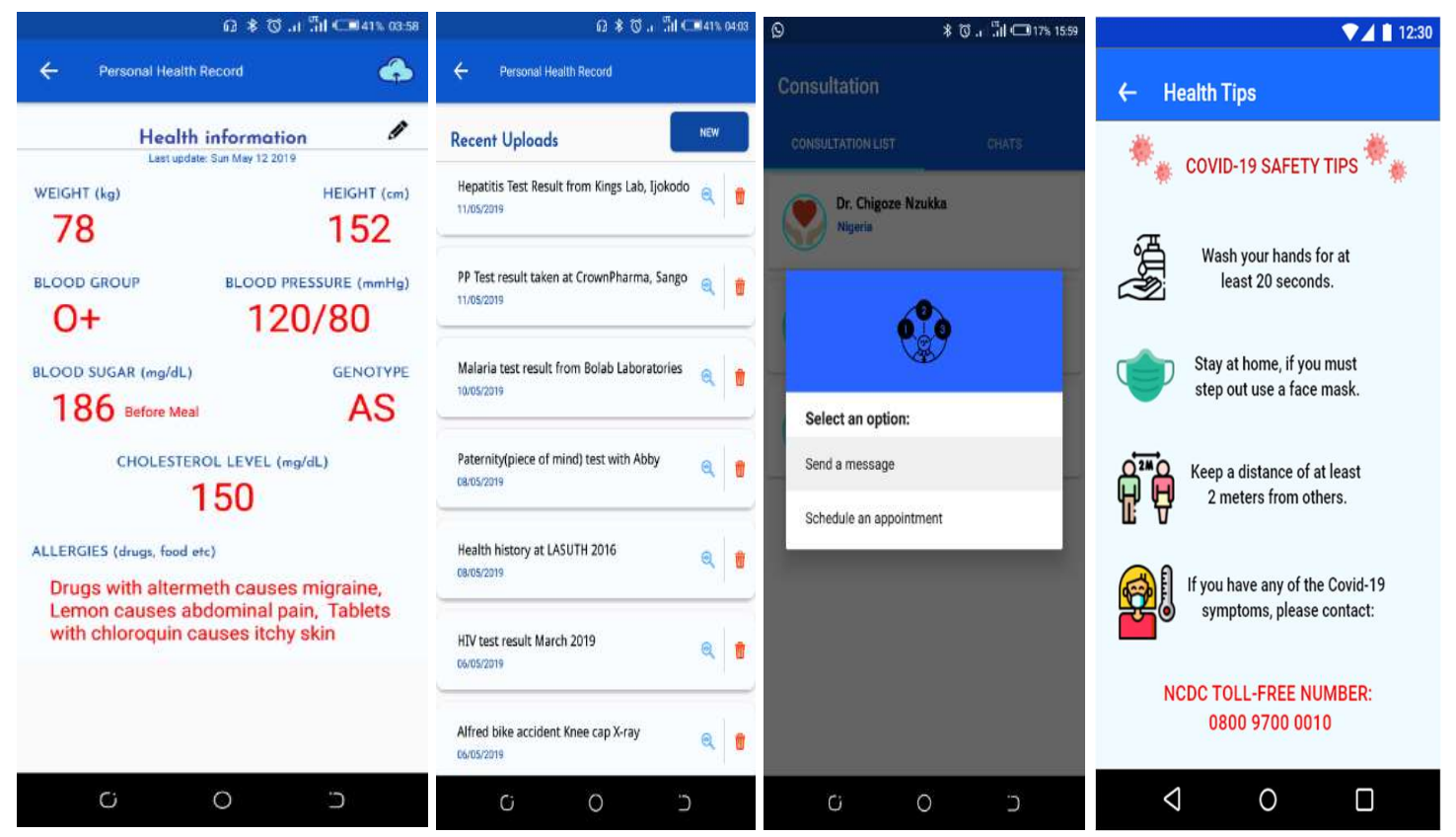

Figure 3: Personal Health Record, Consultation and Appointment and Health Tips Screens of the App

An online questionnaire accompanied the implementation of the application for users to fill after they might have used the application. Six (6) doctors and thirteen (13) patients summed up to a total of nineteen (19) users downloaded, installed and used the app at least once. The following evaluation values were checked during the evaluation. Task Difficulty: The app users were all able to perform account-related tasks easily. $94.7 \%$ of users found it easy to edit their account details. Updating and viewing health information on the app was found easy by $89.6 \%$ of the users. The tasks of reading health tips, instant messaging content upload from phone or camera was relatively easy. However, adding users to the consultation list was found difficult by $21.1 \%$ of the users. 
Perceived Usefulness: Most users of the app found it useful to a large extent. While $94.7 \%$ agree that users can better keep their health record using this app, $94.7 \%$ of the users agree that the application can be of benefit in healthcare and felt confident using it. However, $36.9 \%$ of users need help finding their way efficiently on the app. Performance and Security: All the users experienced a smooth screen transition across the app. The efficient handling of the app activity lifecycle is also reported with no user indicating any app crash. A little above average $(52.6 \%$ ) affirmed that it was easy to store and retrieve data from the app, but $26.3 \%$ disagreed while $21.1 \%$ were undecided. The app is secure as $100 \%$ of the respondents indicated that they needed to provide a correct password before they could access the application.

\section{CONCLUSION AND FUTURE WORK}

The Medipal app is a mobile health application which can be used by doctors and patients for online consultation and appointment scheduling. Users of the application can also keep their background health information and upload health related files. According to the feedback received, the application will be of use in the healthcare industry. This will lead to more managed time and convenience in delivering and receiving healthcare services. With the instant messaging and appointment scheduling feature of the app, doctors can confidentially send messages to their patients and vice-versa, and better manage their time in the physical consultation process. Besides, the app will ensure continuity in healthcare by allowing patients to keep and share their health records across all healthcare centres they visit.

Other modules such as knowledge-based consultation that can help a patient answer some questions which will serve as indicators to determine if a patient is having the symptoms of COVID-19 virus or not can be added. Moreover, a link to data updates from the NCDC and World Health organisation can be added to the app. Future applications can be built based on this study to include features such as a healthcare-centred app, which will enable electronic medical records sent to patients on the app. It can also include a feature containing the list of resident doctors at the healthcare centres with whom patients can consult and schedule appointments.

\section{REFERENCES}

1. Asunmo, A.O. and Yaya, J. A (2016). Management and Preservation of Health Records in some Selected Hospitals in Lagos State, Nigeria. International Journal of Information Research and Review. 3:1601-1605

2. Canadian Medical Association (2015). Guiding Principles for Physicians Recommending Mobile Health Applications to Patients. Retrieved o 3 April 2020 from https://www.cma.ca/sites/default/files/201811/cma_policy_guiding_principles_for_physicians_recommending_mobile_health_applications_to_p atients_pd1-e.pdf

3. Chen, P., Robielos, C., Palana, C., Valencia, L. and Chen. Y. (2015). Scheduling Patients' Appointments: Allocation of Healthcare Services Using Simulation Optimization. Journal of Healthcare Engineering. 6: 259-280.

4. eHealth Network (2020). Mobile applications to support contact tracing in the EU's fight against COVID-19: Common EU Toolbox for Member States, Version 1.0. Retrieved from https://ec.europa.eu/health/sites/health/files/ehealth/docs/covid-19_apps_en.pdf

5. Federal Ministry of Economic Cooperation and Development (2018). Digital Health Ecosystem for African Counties. Retrieved on 10 May 2020 from https://www.bmz.de/en/publications/topics/health/Materilie345_digital_health_africa.pdf 
6. GlobeNewswire (28 April, 2020). The growing penetration for smartphone and internet connectivity in the emerging nation is driving the Mobile Health market. Retrieved from https://www.globenewswire.com/news-release/2020/04/28/2023512/0/en/Mobile-Health-mHealthMarket-To-Reach-USD-311-98-Billion-By-2027-Reports-and-Data.html

7. MacGinnis, G. \& Bennett, F. (2012). Policy and Regulation for Innovation in Mobile Health. Retrieved 23 January 2020, from https://www.gsma.com/mobilefordevelopment/wpcontent/uploads/2012/04/policyandregulationforinnovationinmobilehealth.pdf

8. Newsroom, (2020). Apple and Google partner on COVID-19 Contact Tracing Technology. Retrieved from https://www.apple.com/newsroom/2020/04/apple-and-google-partner-on-covid-19-contact-tracingtechnology/.

9. O'Malley, M. S., Fletcher, S. W., Fletcher, R. H. \& Earp, J. A. (1983). Measuring Patient Waiting Time in a Practice Setting: A Comparison of Methods. Journal of Ambulatory Care Management. 6: 20-27.

10. Obulor, R. \& Eke, B. O. (2016). Outpatient Queuing Model Development for Hospital Appointment System. International Journal of Scientific Engineering and Applied Science. 2(4)

11. Oyegoke, L. (2013). Adoption and Utilization of ICT in Nigeria Hospitals (Government Owned). Bachelor's Thesis. Haaga-Helia University of Applied Sciences, Finland.

12. Salami, O. O. (2015). Privacy Protection for Mobile Health (mhealth) in Nigeria: A Consideration of the EU Regime for Data Protection as a Conceptual Framework for Reforming Nigeria's Privacy Legislation. Unpublished M.Sc. Thesis, Dalhouise University, Canada. Retrieved 20 July, 2018, from http://dalspace.library.dal.ca/handle/10222/56333

13. Senkovska, Z. (26 October, 2017). Building a Medical App for Physicians and Patients. Retrieved June 6, 2018, from https://apiko.com/blog/building-a-medical-app-for-physicians-and-patientsfactsexamples-and-techniques-2/

14. Stevenson, D. (2018). What is Firebase? The Complete Story, abridged. [Web blog post]. Retrieved March 10, 2019, from https://blog.usejournal.com/what-is-firebase-thecomplete-story-abridgedbcc730c5f2c0

15. United Nations Foundation (UNF) (2014). Assessing the Enabling Environment for ICTs for Health in Nigeria: A Review of Policies.

16. Ventola, C. L. (2014). Mobile Devices and Apps for Health Care Professionals: Uses and Benefits. Journal for Managed Care and Hospital Formulary Management, 39(5): 356-364.

17. World Health Organisation (2011). mHealth: New Horizons for Health Through Mobile Technologies. Global Observatory for eHealth Series - Volume 3. Retrieved on 22 June 2020 from https://www.who.int/goe/publications/goe_mhealth_web.pdf?

18. World Health Organisation (2020). Support to Health Workers on COVID-19 through a WHO App. Assessed from https://www.who.int/news-room/feature-stories/detail/support-to-health-workers-oncovid-19-through-a-who-app 